\title{
Béatrice Damian-Gaillard, Sandy Montañola et Aurélie Olivesi (dir.) : L'assignation de genre dans les médias. Attentes, perturbations, reconfigurations
}

\section{Sarah Lécossais}

\section{(2) OpenEdition}

\section{Journals}

Édition électronique

URL : http://journals.openedition.org/edc/6140

DOI : $10.4000 /$ edc. 6140

ISSN : 2101-0366

Éditeur

Université de Lille

\section{Édition imprimée}

Date de publication : 1 juin 2015

Pagination : 178-181

ISBN : 978-2-917562-13-0

ISSN : $1270-6841$

Référence électronique

Sarah Lécossais, « Béatrice Damian-Gaillard, Sandy Montañola et Aurélie Olivesi (dir.) : L'assignation de genre dans les médias. Attentes, perturbations, reconfigurations ", Études de communication [En ligne], 44 2015, mis en ligne le 02 juin 2015, consulté le 22 septembre 2020. URL : http:// journals.openedition.org/edc/6140; DOI : https://doi.org/10.4000/edc.6140

Ce document a été généré automatiquement le 22 septembre 2020.

(c) Tous droits réservés 


\title{
Béatrice Damian-Gaillard, Sandy Montañola et Aurélie Olivesi (dir.) : L'assignation de genre dans les médias. Attentes, perturbations, reconfigurations
}

\author{
Sarah Lécossais
}

\section{RÉFÉRENCE}

Béatrice Damian-Gaillard, Sandy Montañola et Aurélie Olivesi (dir.) : L'assignation de genre dans les médias. Attentes, perturbations, reconfigurations, Rennes, Presses universitaires de Rennes, 2014, 154 p.

Ouvrage collectif dirigé par Béatrice Damian-Gaillard, Sandy Montañola et Aurélie Olivesi, L'assignation de genre dans les médias. Attentes, perturbations, reconfigurations propose d'explorer les représentations sociales du genre dans divers contenus médiatiques, allant de la presse (magazine ou d'information, nationale et internationale) à la téléréalité en passant par la publicité et les séries télévisées. Dans l'introduction, les directrices de l'ouvrage conçoivent les discours médiatiques en tant qu'ils sont structurés par les représentations sociales tout autant qu'ils participent à les configurer. En ce sens, ces discours s'appuient sur la mise en scène de catégories sexuées, que les stéréotypes vont ancrer dans des normes et des systèmes de croyances relatifs à ce que seraient le féminin et le masculin. Elles proposent alors la définition suivante de l'assignation de genre: "l'action d'assigner consiste à attribuer à une personne une place, une fonction, un rôle, et plus particulièrement, attendre qu'elle le performe en se conformant aux attentes sociales construites autour des identités de genre, selon qu'elle est perçue comme étant un homme ou une femme" (p.13). Le recours à cette notion permet d'explorer - en reprenant la catégorisation tripartite de 
Ruth Amossy ${ }^{1}$ - «les attentes envers le genre, les perturbations, puis les recatégorisations de ces attentes » (p.15). C'est d'ailleurs cette catégorisation qui guide l'organisation en trois parties de l'ouvrage, composées chacune de trois chapitres.

La première partie questionne l'actualisation par les médias des attentes normatives liées au genre et s'ouvre sur le travail d'Élise Vinet et Stéphanie Gosset sur les représentations de l'allaitement dans la presse parentale française. Les normes véhiculées ne concernent pas tant le mode d'allaitement - au sein ou au biberon - que les « rôles de sexe ». Ainsi, ce qui compte, dans ce corpus, c'est que ce soient les femmes qui allaitent - de quelque manière que ce soit -, ce qui témoigne d'un renforcement des représentations traditionnelles et stéréotypées des rôles sociaux de sexe, tout comme d'une assignation très forte des femmes au care et à la prise en charge des enfants. Laetitia Biscarrat, dans le deuxième chapitre, analyse la figure de la mère célibataire dans un programme de téléréalité centré sur la quête amoureuse. L'auteure montre que seuls les personnages se conformant aux attentes normatives liées à leur genre parviennent à trouver un partenaire. La mère célibataire " acceptable » est donc tout d'abord celle qui multiplie les rôles sociaux - elle doit être mère mais aussi épouse/ amante, sans pour autant surinvestir l'un de ces rôles au détriment de l'autre. De plus, elle doit accepter la hiérarchisation entre les genres - ne pouvant de ce fait être ouvertement féministe -, et répondre de manière positive à l'implicite blanc d'un « citoyen français idéal »- les non-blancs étant ethnoracialisés. C'est d'ailleurs à cette co-construction des rapports sociaux de genre, de race et de classe que s'intéresse également Marion Dalibert. Celle-ci déconstruit dans son article les divers processus d'altérisation des non-blancs dans la couverture médiatique (presse quotidienne nationale dite de référence et journaux télévisés) de Ni putes ni soumises. Sont ici mis en lumière trois duos de stéréotypes (la "jeune fille» et le «jeune garçon des banlieues " par exemple), au sein desquels race et genre sont intrinsèquement liés. Ces stéréotypes sont « catégorisés et ethnoracialisés par la représentation d'une identité de genre déviante à l'égard des normes françaises de masculinité et de féminité » (p. 62). À travers ces duos, c'est en réalité une frontière entre «blancs » et «non-blancs » qui est dessinée, révélant, en creux, un sujet de la nation répondant à des attentes normatives en termes de race, de classe, et même de sexualité.

3 Si dans la première partie ce sont les attentes normatives liées au genre qui sont étudiées, les trois chapitres suivant s'intéressent aux décrochements ou décalages visà-vis de ces attentes. Angela Merkel et les femmes terroristes, respectivement étudiées par Juliette Charbonneaux et Fanny Bugnon dans des corpus de presse, sont des exemples tout à fait révélateurs de ces perturbations. La première n'est pas uniquement construite comme "femme ", mais est caractérisée par une "ronde des assignations» (p.76) qui témoigne du poids de la religion, de la situation professionnelle ou encore de l'origine géographique dans la représentation des identités de genre. Les terroristes viennent à leur tour brouiller les normes de genre, tant leur violence ou l'absence de maternité - sur lesquelles les journalistes insistent rompent avec les assignations traditionnelles. Si les stéréotypes de genre sont bousculés, on assiste en contrepoint à une "volonté quasi consensuelle de réaffirmation de l'ordre des sexes »(p.90). Cette partie se clôt sur une réflexion méthodologique de Sarah Sepulchre à partir d'un corpus de séries télévisées. La chercheuse met en lumière le risque inhérent à l'analyse de ce genre de corpus en termes de stéréotypage, à savoir la réaffirmation de la catégorisation sexuée des 
identités. Comment analyser les stéréotypes de genre sans recourir à l'emploi de termes eux-mêmes genrés, voire stéréotypés?

4 La dernière partie de l'ouvrage est consacrée aux reconfigurations des attentes normatives liées au genre. Deux chapitres traitent de la publicité et de l'hyperritualisation du genre qui s'y déploie. Dans le premier cas, analysé par Jean-Claude Soulages, une configuration hégémonique - caractérisée par la stabilité des représentations clivées - est concurrencée par une posture contre-hégémonique mettant en avant de nouvelles identités narratives, notamment féminines. Cependant, un conservatisme persistant reconduit des assignations genrées dans les représentations publicitaires du travail ou de la sphère domestique par exemple. Le chapitre de Stéphanie Kunert montre que si les personnages gays, travestis ou transexuels « ont valeur d'écart par rapport à une conception du genre [...] et de la sexualité (comme hétérosexuelle) que la publicité désigne comme la normalité » dans les publicités où les minorités sexuelles ne sont pas destinataires, leur représentation change lorsqu'ils deviennent destinataires via un phénomène de normalisation de l'homosexualité (dans le cadre du gay marketing). Finalement, chez cette auteure comme chez Jean-Claude Soulages, le défigement des identités genrées vient en réalité réaffirmer la norme en la réifiant. Ainsi, les perturbations ne suffisent pas à modifier les représentations et se heurtent au "plafond de verre du prêt à penser " (Soulages, p.121). Dans le dernier chapitre, Gabrielle Trépanier-Jobin s'interroge sur la capacité de la parodie à transgresser les stéréotypes de genre dans le feuilleton télévisé satirique Le coeur a ses raisons et conclut que la "parodie n'est pas un moyen de subversion sans risque » (p. 151). Si celle-ci autorise des procédés de dénaturalisation des stéréotypes de genre, elle échoue à proposer de nouveaux modèles.

Nous pouvons tout d'abord relever l'existence d'un socle commun à la plupart de ces textes sur l'assignation de genre dans les médias, s'appuyant régulièrement sur les travaux d'Erving Goffman, Teresa de Lauretis, Marlène Coulomb-Gully ou Marie-Joseph Bertini sur le genre et ses représentations médiatiques. Cet ouvrage insiste également sur la nécessité et sur la pertinence heuristique de penser le genre en regard de la race, de la religion, de la sexualité, de la classe ou encore de l'origine géographique. L'une des grandes qualités de ces chapitres est ainsi la perspective intersectionnelle qui y est adoptée. Par ailleurs, l'analyse de ces différents corpus rend compte d'une disqualification régulière du féminisme et de ses acquis. L'invalidation de la critique féministe prend forme à travers divers processus: association du féminisme à une conduite passionnée et outrancière chez les mères célibataires, à la violence du côté des terroristes ou encore à la défense d'intérêts de classe et de race à travers la figure stéréotypique de la "féministe blanche ». Enfin, si l'ouvrage montre que les procédés d'assignation prennent des formes multiples, il témoigne en même temps des résistances, transgressions ou reconfigurations possibles. Cependant, le constat demeure amer, au terme de cette lecture, car, que les attentes liées au genre soient performées, perturbées ou reconfigurées, la norme finit toujours, d'une manière ou d'une autre, par être réaffirmée. 


\section{NOTES}

1. Amossy Ruth (1991), Les idées reçues. Sémiologie du stéréotype, Paris, Nathan, 215 p.

\section{AUTEUR}

\section{SARAH LÉCOSSAIS}

Laboratoire CIM, équipe MCPN - Université Sorbonne Nouvelle - Paris 3 